\title{
Visual features that vary together over time group together over space
}

David Alais, Randolph Blake and Sang-Hun Lee

Nature Neurosci. 1, 160-164 (1998).

The following paragraphs were inadvertently omitted from the results section. The end of that section should read:

(page 162)...More importantly, correlated contrast modulation still produced greater coherence compared to the uncorrelated case even when the two gratings differed in contrast.

Contrast modulation of a drifting grating introduces additional temporal frequencies in the stimulus, besides those associated with the smooth drift rate. Because the contrast modulations were purposefully random, the fluctuations in temporal frequency were also random. In the case of our two-component plaid, random contrast modulations effectively modulate the length (but not the direction) of the component vectors, and these random fluctuations in vector length could be perceived as irregularities in speed, or motion 'jerkiness.' For uncorrelated contrast modulations, the changes in vector length would differ for the two components and, therefore, possibly complicate the grouping process. But why would irregular, jerky motion that is correlated among components increase perceived coherence, the result we obtained? One might argue that correlated jerkiness leads the visual system intelligently to deduce that the jerky components belong to the same object.

To test this possibility, we created a plaid consisting of two components that did not modulate in contrast. In two conditions, we explicitly selected a new drift-rate every $40 \mathrm{~ms}$ from a set of seven ranging from $4-8 \mathrm{~Hz}$; this caused motion to appear jerky. In one of these conditions, the random fluctuations in drift-rate were correlated, and in the other they were uncorrelated. In a third condition, the component gratings drifted smoothly at a constant rate of $6 \mathrm{~Hz}$ throughout the observation period. Observers again tracked periods of global coherence during 60-second observation periods. Jerky motion had no effect on perceived coherence, which was equivalent for all three conditions. Accordingly, we conclude that jerkiness is not responsible for the increased coherence produced by correlated contrast modulations. 